\title{
Determinación de la velocidad específica de absorción (SAR) en sistemas WiFi y Wi-Max mediante un modelo bioplasmático quiral
}

\author{
Carina C. García(1) y Mario L. Zamorano(2) \\ (1) Universidad de Tarapacá, Programa de Magister en Telecom Casilla 6-D, Arica, Chile. \\ (correo-e: cgarcia.uta@gmail.com) \\ (2) Universidad de Tarapacá, Escuela de Ingeniería Eléctrica-Electrónica, Casilla 6-D, Arica, Chile. \\ (correo-e: mhzlucero@uta.cl)
}

Recibido Ago. 6, 2019; Aceptado Sep. 30, 2019; Versión final Nov. 29, 2019, Publicado Abr. 2020

\section{Resumen}

Se presenta un modelo bioplasmático-quiral que caracteriza la cabeza humana y analiza su comportamiento cuando es radiada por las microondas de sistemas inalámbricos. La técnica del método de las diferencias finitas en el dominio del tiempo permite calcular numéricamente los campos electromagnéticos y simular la tasa de absorción especifica. Los resultados se obtuvieron para el corte 35 del modelo de imágenes de resonancia magnética. La conclusión más importante de nuestro trabajo es que, considerando que el tejido cerebral es un bioplasma quiral, se produce mayor absorción, respecto de los modelos clásicos, de la radiación de microonda que emiten los sistemas WiFi y Wi-Max.

Palabras clave: velocidad específica de absorción; SAR; WiFi; Wi-Max; modelo quiral

\section{Determination of the specific absorption rate (SAR) in WiFi and Wi-Max systems by means of a chiral bioplasma model}

\begin{abstract}
A chiral-bioplasma model that characterizes the human head and analyzes its behavior when it is radiated by the microwave of wireless systems is presented. The technique of the method of finite differences in the domain of time allows numerical calculation of electromagnetic fields and simulates the specific absorption rate. The results were obtained for section 35 of the magnetic resonance imaging model. The most important conclusion of our work is that, considering that the brain tissue is a chiral bioplasma, there is greater absorption, compared to the classic models, of the microwave radiation emitted by the WiFi and Wi-Max systems.
\end{abstract}




\section{INTRODUCCIÓN}

El uso de tecnología digital inalámbrica ha crecido exponencialmente durante las últimas décadas (ITU-D, 2016). Los sistemas inalámbricos y móviles utilizan radiación de radiofrecuencia (RF) no ionizante en el rango de frecuencias de $30 \mathrm{KHz}$ a $300 \mathrm{GHz}$. Con este aumento a la exposición de las microondas de los sistemas móviles, también aumenta la preocupación por los posibles daños a la salud de los usuarios al utilizar estos dispositivos. Por este motivo se han realizado una gran cantidad de estudios al respecto, entre los últimos podemos citar a Cardis et al. (2010), Sage (2012) y Wyde et al., (2016). Además, en mayo de 2011 la Agencia Internacional para la Investigación del Cáncer (AllC), de la Organización Mundial de la Salud (OMS), realizó una evaluación científica sobre el riesgo de tumor cerebral debido al uso de teléfonos móviles, que permitiera determinar el potencial riesgo carcinógeno debido a la exposición de la RF. Como resultado de la reunión se incorporó, en la clasificación de la AllC y OMS, a los campos electromagnéticos de RF emitidos por los dispositivos de telefonía inalámbrica como posiblemente carcinógenos para los humanos, grupo 2B, WHO/AIRC (2018). La clasificación incluye todas las fuentes de radiación de RF: estaciones bases de telefonía móvil, WiFi, Wi-Max, teléfonos inteligentes, computadoras portátiles, tabletas, etc. Toda esta información mantiene la preocupación del riesgo de desarrollar tumores, como resultado de la exposición a las radiaciones de RF, lo que puede tener graves consecuencias para la salud pública debido a que el uso generalizado de la ubicua telefonía celular y de los accesos inalámbricos a Internet forman una parte muy importante en la vida de las personas, de los hogares y de los lugares de trabajo.

La preocupación surge del hecho que el cuerpo de los usuarios de la tecnología inalámbrica, particularmente la cabeza humana, en el caso de los teléfonos celulares, está sometido a una exposición localizada de radiación de microondas y por lo tanto estos campos electromagnéticos penetran los tejidos expuestos y absorben la radiación. El cerebro es uno de los órganos al que más afecta la radiación de microondas, lo que aún es más grave en el caso que los usuarios de los sistemas inalámbricos sean niños o adolescentes, por ser personas en desarrollo (Feychting, 2011; Chiu et al., 2015; Gandhi 2015; Cho y Lee, 2017). Aunque existe gran cantidad de trabajos en el área, aún no hay un conocimiento suficientemente completo sobre este tema en particular. Sin embargo, existe un consenso general acerca de la importancia de evaluar correctamente los mecanismos de interacción entre los campos electromagnéticos y los sistemas biológicos (Lin, 2009), (Baliatsas et al., 2012; Ferikoğlu et al., 2014; González-Rubio et al., 2017; Rashed et al., 2019; Miyakoshi et al., 2019). Los diferentes modelos de cabeza humana van desde modelos simples, como una esfera homogénea, hasta modelos heterogéneos, anatómicamente correctos basados en imágenes de resonancia magnética (MRI: Magnetic Resonance Image), (Makris et al., 2008). El método de las diferencias finitas en el dominio del tiempo (FDTD: Finite Difference Time Domain) se ha utilizado ampliamente para dosimetría bioelectromagnética (Martínez et al., 2004; Morris et al., 2015; Krayni et al., 2016).

El estudio de los efectos térmicos y no térmicos que causa la radiación celular sobre la cabeza humana requiere analizar en detalle la absorción fundamental, de manera de obtener una indicación básica del coeficiente de absorción específica, SAR (Moore et al., 2015; Chiaramello et al., 2019). Por ello, después de definir el modelo de cabeza a utilizar, se debe analizar en detalle la absorción fundamental producida por la radiación celular sobre los tejidos internos que componen la cabeza humana, para lo cual se requiere evaluar los CEM incidentes. En este artículo se presenta un modelo basado en el método FDTD multigrid que considera el comportamiento neurológico del tejido cerebral bajo radiación de microondas, particularmente del WiFi y WiMax, por la importancia que tienen en los sistemas inalámbricos actuales. En base a nuestra experiencia anterior, usamos el modelo quiro-electrodinámico simple, que tiene en cuenta la interacción principal entre el bioplasma de las neuronas cerebrales y la radiación de microondas de los sistemas (Torres y Zamorano 2003; Torres y Zamorano 2005). Luego se explica la teoría de la técnica utilizada y la descripción del modelo, después se presentan y analizan los resultados obtenidos para el SAR en la capa 35. Finalmente, se presentan las conclusiones.

\section{FUNDAMENTOS Y MODELOS}

Nuestro modelo se basa en el hecho de que el tejido cerebral está formado por neuronas. Las neuronas del cerebro están organizadas por redes integradas de polímeros de proteínas llamadas citoesqueleto (Hameroff y Penrose, 1996). El citoesqueleto se compone de microtúbulos, los que son cilindros huecos, de $25 \mathrm{~nm}$ de diámetro, cuyas longitudes varían y puede ser bastante largas dentro de algunos axones nerviosos (ver Fig. 1). Las paredes del cilindro de los microtúbulos están compuestas por 13 protofilamentos longitudinales, los que son, cada uno de ellos, una serie de proteínas de subunidades conocidas como tubulinas, en cuya estructura se hallan dos subunidades denominadas dímeros $\alpha$ y $\beta$, ensambladas helicoidalmente, las cuales pueden tener las configuraciones que se muestran en la Figura 2. Nuestra hipótesis plantea que estas configuraciones corresponden a la quiralidad hacia la izquierda y hacia la derecha respectivamente, ya que ellas representan los estados de polarización de los dímeros eléctricos. 


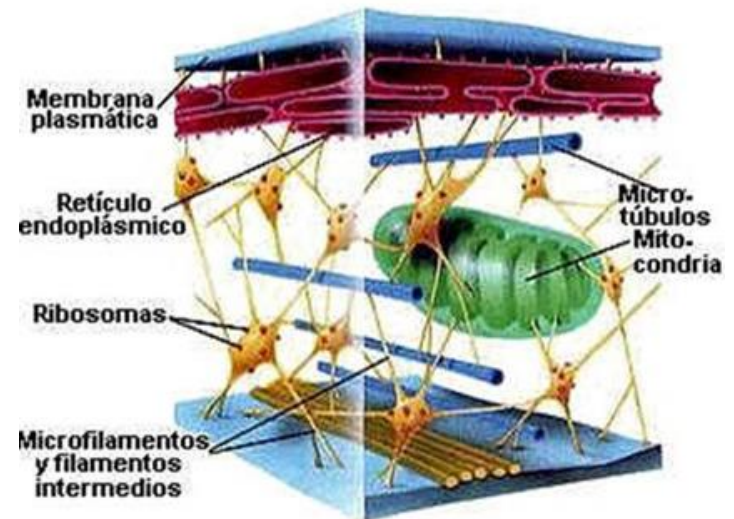

Fig. 1: Estructura de la neurona (Datos tomados de Torres y Zamorano, 2003)

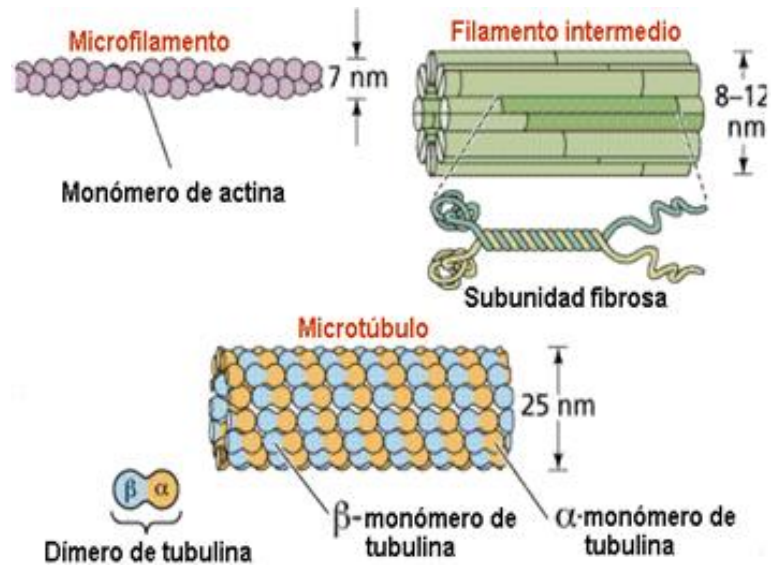

Fig. 2: Microtúbulos en tubulinas $\alpha$ y $\beta$ (Datos tomados de Torres y Zamorano, 2003)

Entonces, dado que el medio de la proteína es quiral, una onda electromagnética en ese medio rotará necesariamente su plano de polarización de acuerdo con la estructura biológica dominante. Cálculos preliminares demuestran que los valores propios de la frecuencia de estas proteínas están entre 1 y $10 \mathrm{GHz}$. Estas frecuencias son típicas de la radiación de microondas, lo que corresponde a oscilación coherente dentro del microtúbulo. De acuerdo con el planteamiento anterior, el modelo quiro-electrodinámico sugiere usar técnicas estándar no solo para caracterizar la radiación electromagnética-tejido biológico (MRI, FDTD, SAR), sino también para demostrar que el tejido cerebral es un bioplasma quiral y, por lo tanto, nosotros aplicamos las ecuaciones de Maxwell a un conjunto de microtúbulos y moléculas helicoidales embebidas en un sustrato, caracterizadas por un factor de quiralidad global, dado por el factor T (ver ecuaciones 1 y 2 ).

Los medios quirales responden a la excitación tanto de campos magnéticos como eléctricos (Espinosa et al., 2011). Por lo tanto, pertenecen a la clase general de medios bianisotrópicos de comunicación. Estos medios pueden caracterizarse por un conjunto generalizado de relaciones constitutivas en las que los campos eléctricos y magnéticos están acoplados. Existen diferentes expresiones para las relaciones constitutivas. En este artículo consideramos las ecuaciones constitutivas de Born-Fedorov:

$$
\begin{aligned}
& \mathrm{D}=\varepsilon(\mathrm{E}+\mathrm{T} \nabla \times \mathrm{E}) \\
& \mathrm{B}=\mu(\mathrm{H}+\mathrm{T} \nabla \times \mathrm{H})
\end{aligned}
$$

donde, $\varepsilon, \mu$ y $\mathrm{T}$ son la permitividad, la permeabilidad y el factor quiral respectivamente. Este último representa la medida de la quiralidad y tiene unidades de longitud. Si se asume que el medio es isótropo, impermeable y no dispersivo, las componentes cartesianas del campo son:

$$
\begin{aligned}
& \mathrm{D}_{\mathrm{x}, \mathrm{y}, \mathrm{z}}=\varepsilon_{0} \varepsilon_{\mathrm{r}} \mathrm{E}_{\mathrm{x}, \mathrm{y}, \mathrm{z}}+\varepsilon_{0} \varepsilon_{\mathrm{r}} \mathrm{T}\left[\frac{\partial \mathrm{E}_{\mathrm{z}, \mathrm{x}, \mathrm{y}}}{\partial \mathrm{y}, \mathrm{z}, \mathrm{x}}-\frac{\partial \mathrm{E}_{\mathrm{y}, \mathrm{z}, \mathrm{x}}}{\partial \mathrm{z}, \mathrm{x}, \mathrm{y}}\right] \\
& \mathrm{B}_{\mathrm{x}, \mathrm{y}, \mathrm{z}}=\mu_{0} \mathrm{H}_{\mathrm{x}, \mathrm{y}, \mathrm{z}}+\mu_{0} \mathrm{~T}\left[\frac{\partial \mathrm{H}_{\mathrm{z}, \mathrm{x}, \mathrm{y}}}{\partial \mathrm{y}, \mathrm{z}, \mathrm{x}}-\frac{\partial \mathrm{H}_{\mathrm{y}, \mathrm{z}, \mathrm{x}}}{\partial \mathrm{z}, \mathrm{x}, \mathrm{y}}\right]
\end{aligned}
$$


Usando el sistema de unidades MKS, las ecuaciones escalares, que se presentan a continuación, son equivalentes a las ecuaciones de Maxwell en el sistema de coordenadas rectangulares $(x, y, z)$ :

$$
\begin{aligned}
& \frac{\partial \mathrm{H}_{\mathrm{x}, \mathrm{y}}}{\partial \mathrm{t}}=\mp \frac{1}{\mu} \frac{\partial \mathrm{E}_{\mathrm{z}}}{\partial \mathrm{y}, \mathrm{x}} \pm \mathrm{T} \omega \mathrm{k}_{\mathrm{y}, \mathrm{x}} \mathrm{H}_{\mathrm{z}} \\
& \frac{\partial H_{z}}{\partial t}=\frac{1}{\mu}\left(\frac{\partial E_{x}}{\partial y}-\frac{\partial E_{y}}{\partial x}\right)+\mathrm{T} \omega\left(k_{x} H_{y}-k_{y} H_{x}\right) \\
& \frac{\partial \mathrm{E}_{\mathrm{x}, \mathrm{y}}}{\partial \mathrm{t}}= \pm \frac{1}{\varepsilon} \frac{\partial \mathrm{H}_{\mathrm{z}}}{\partial \mathrm{y}, \mathrm{x}} \pm \mathrm{T} \omega \mathrm{k}_{\mathrm{y}, \mathrm{x}} \mathrm{E}_{\mathrm{z}}-\sigma \mathrm{E}_{\mathrm{x}, \mathrm{y}} \\
& \frac{\partial \mathrm{E}_{\mathrm{z}}}{\partial \mathrm{t}}=\frac{1}{\varepsilon}\left(\frac{\partial \mathrm{H}_{\mathrm{y}}}{\partial \mathrm{x}}-\frac{\partial \mathrm{H}_{\mathrm{x}}}{\partial \mathrm{y}}\right)+\mathrm{T} \omega\left(\mathrm{k}_{\mathrm{x}} \mathrm{E}_{\mathrm{y}}-\mathrm{k}_{\mathrm{y}} \mathrm{E}_{\mathrm{x}}\right)-\sigma \mathrm{E}_{\mathrm{z}}
\end{aligned}
$$

Donde $\omega=2 \pi f ; k_{x}=2 \pi / \lambda_{x} ; k_{y}=2 \pi / \lambda_{y} y \sigma\left(S m^{-1}\right)$ es la conductividad eléctrica del tejido.

En el sistema de ecuaciones diferenciales presentado, se observa que las componentes vectoriales del flujo eléctrico están relacionadas con las respectivas componentes del campo eléctrico y además son proporcionales a las derivadas parciales de sus componentes ortogonales. La dificultad evidente de un tratamiento analítico de estas ecuaciones radica en que poseen derivadas parciales espacio-temporales, donde interviene la quiralidad del medio, lo que impide que puedan ser reducidas a una ecuación diferencial tradicional cuya solución sea conocida y es necesario, por lo tanto, recurrir a un tratamiento numérico.

El método FDTD, propuesto inicialmente por Yee, se usa comúnmente en la solución de las ecuaciones de Maxwell. A través del método FDTD, se realiza la discretización de las ecuaciones anteriores. En nuestra formulación, se utiliza la aproximación de segundo orden de Mur para los problemas de radiación de campo cercano en un caso de interfaz aquiral-quiral (Taflove y Hagness, 2005). Después del cálculo del campo eléctrico quiral inducido, por el método FDTD, la tasa de absorción específica SAR se calcula como:

$$
S A R_{i, j}=\frac{\left.\sigma_{i, j} E_{T}^{2}\right|_{i, j}}{2 \rho_{i, j}}
$$

Donde $\rho$ es la densidad de la muestra de tejido biológico, y

$$
\left.E_{T}\right|_{i, j}=\sqrt{\left.\frac{1}{n} \sum_{1}^{n} E_{y}^{2}\right|_{i, j} ^{n}+\left.E_{x}^{2}\right|_{i, j} ^{n}+\left.E_{z}^{2}\right|_{i, j} ^{n}}
$$

Las etapas de cálculo del modelo bioplasmático para la determinación de SAR se muestran en la Figura 3. EI método consta de los siguientes pasos principales: (1) Modelado de la cabeza humana a través de imágenes MRI, (2) Evaluación de la distribución electromagnética dentro del objetivo biológico, considerando el tejido cerebral como medio bioplasmático quiral, y (3) Simulación SAR para evaluar el efecto térmico.

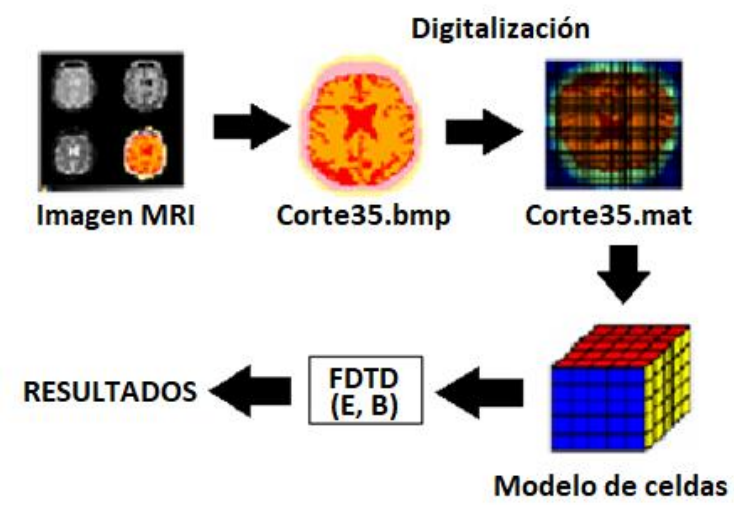

Fig. 3: Diagrama en bloque del proceso para el cálculo del coeficiente SAR (Adaptada de Torres y Zamorano, 2005). 


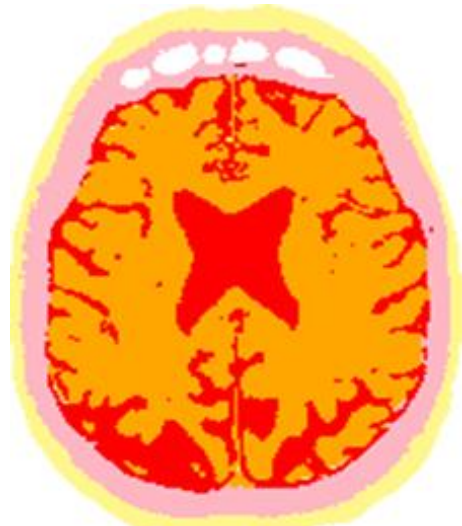

Fig. 4: Imagen del corte 35 (Datos tomados de Torres y Zamorano, 2005).

\section{SIMULACIÓN SAR Y ANÁLISIS DE RESULTADOS}

Para las simulaciones del SAR se usaron la plataforma Matlab y el algoritmo FDTD con quiralidad, este último obtenido de las ecuaciones (5) - (10). Los resultados logrados corresponden a los sistemas Wi-Fi operando en $2,4 \mathrm{GHz}$ y Wi-Max, operando en 5,2 y $5,8 \mathrm{GHz}$. Las simulaciones se realizaron de manera sistemática, para determinar el efecto que la variación del factor quiral del modelo bioplasmático tuvo sobre el coeficiente de absorción, SAR. Estos resultados corrigen resultados anteriores en las frecuencias de 2,4 y $5,2 \mathrm{GHz}$ (Torres y Zamorano 2005), en cambio para la frecuencia de $5,8 \mathrm{GHz}$, correspondiente al sistema WiMax, los resultados obtenidos con el modelo bioplasmático se presentan por primera vez.

El modelo de cabeza humana se construyó con 540000 células cúbicas de 2,5 mm de lado cada una. El número total de capas, contadas desde la parte inferior de la cabeza, utilizadas en este modelo fue 54. Aquí elegimos la capa 35, Figura 4, porque tiene una gran concentración de tejido cerebral. Se consideran cuatro tipos de tejidos (piel, hueso, sangre y cerebro). Para estudiar el efecto quiral, se realizaron cálculos para el supuesto de onda plana, se consideró una potencia $125 \mathrm{~mW}$, teniendo la antena del teléfono celular una impedancia de 180 ohms. Las constantes dieléctricas y las conductividades de los medios considerados se obtuvieron de la literatura (Torres y Zamorano 2005).

Los resultados, para la capa 35, se muestran en las figuras 5-7. Debido a las diferentes propiedades electromagnéticas de los tejidos, la absorción de potencia no es una función de la profundidad que disminuya monótonamente. Para $\mathrm{kT}=0$, la absorción es más alta en la piel, baja en el cráneo, pero más alta nuevamente en el cerebro. Estas curvas se obtuvieron realizando simulaciones similares y luego obteniendo un promedio estadístico del SAR. En todas las curvas, a medida que la onda penetra en la cabeza, atraviesa las diferentes partes de las cuales está hecho el modelo de cabeza y el valor del SAR se atenúa rápidamente debido al cambio en el medio y al aumento de la distancia desde la fuente emisora (antena). Es importante decir que el SAR máximo (punto caliente) está en la parte externa (piel). Después de pasar a través del nivel del hueso, el SAR se atenúa mucho (entre las células 5 y 10) y alrededor de la célula 10 el SAR aumenta a nivel del cerebro. Aquí, a partir de la celda 10 aproximadamente, es importante analizar el rendimiento de nuestro modelo bioplasmático.

La Figura 5 muestra la distribución del SAR para la capa 35, en función de la distancia, para diferentes valores del factor quiral cuando la frecuencia de trabajo es $2.4 \mathrm{GHz}$. EI SAR máximo encontrado, en la región hematoencefálica, fue $0,09 \mathrm{~W} \mathrm{kg-1}$ para $\mathrm{kT}=0$ y $0,13 \mathrm{~W} \mathrm{kg-1}$ para $\mathrm{kT}=1$, es decir, un aumento del $44 \%$ con respecto al caso aquiral. Para un valor de factor quiral igual a 2, el SAR máximo fue $0,15 \mathrm{~W} \mathrm{~kg}-1$, es decir, un aumento del $66 \%$ con respecto al caso de quiralidad nula. Para un valor alto de factor quiral, $\mathrm{kT}=3$, el SAR máximo fue $0.16 \mathrm{~W} \mathrm{~kg}-1$ con un aumento del $77 \%$.

La Figura 6 muestra el SAR para las mismas condiciones anteriores, pero a una frecuencia de $5,2 \mathrm{GHz}$. El SAR máximo encontrado, en la región hematoencefálica, fue $0,5 \mathrm{~W} \mathrm{kg-1}$ para $\mathrm{kT}=0$ y $0,65 \mathrm{~W} \mathrm{~kg}-1$ para $\mathrm{kT}=$ 1 , es decir, un aumento del $30 \%$ con respecto al caso $\mathrm{kT}=0$. Para un valor de factor quiral $\mathrm{kT}=2$, el $\mathrm{SAR}$ máximo fue $0,77 \mathrm{~W} \mathrm{~kg}-1$, un aumento del $54 \%$ con respecto al caso de quiralidad nula. Para el factor quiral, $\mathrm{kT}=3$, el SAR máximo fue $0.79 \mathrm{~W} \mathrm{kg-1}$ con un aumento del $58 \%$. En la figura 7 , para la frecuencia de 5,8 $\mathrm{GHz}$, el SAR máximo encontrado, en la región hematoencefálica, fue 0,6 $\mathrm{W} \mathrm{kg-1}$ para $\mathrm{kT}=0$ y $0,8 \mathrm{~W} \mathrm{~kg}-1$ para $\mathrm{kT}=1$, es decir, en este caso se tuvo un aumento del $33 \%$ con respecto al caso aquiral. Para $\mathrm{kT}=2$, el SAR máximo fue $0.9 \mathrm{~W} \mathrm{kg-1}$, lo que significa que el aumento fue el $58 \%$. Para $\mathrm{kT}=3$, el SAR máximo fue $0.95 \mathrm{~W} \mathrm{~kg}-1$ con un aumento del $58 \%$. 


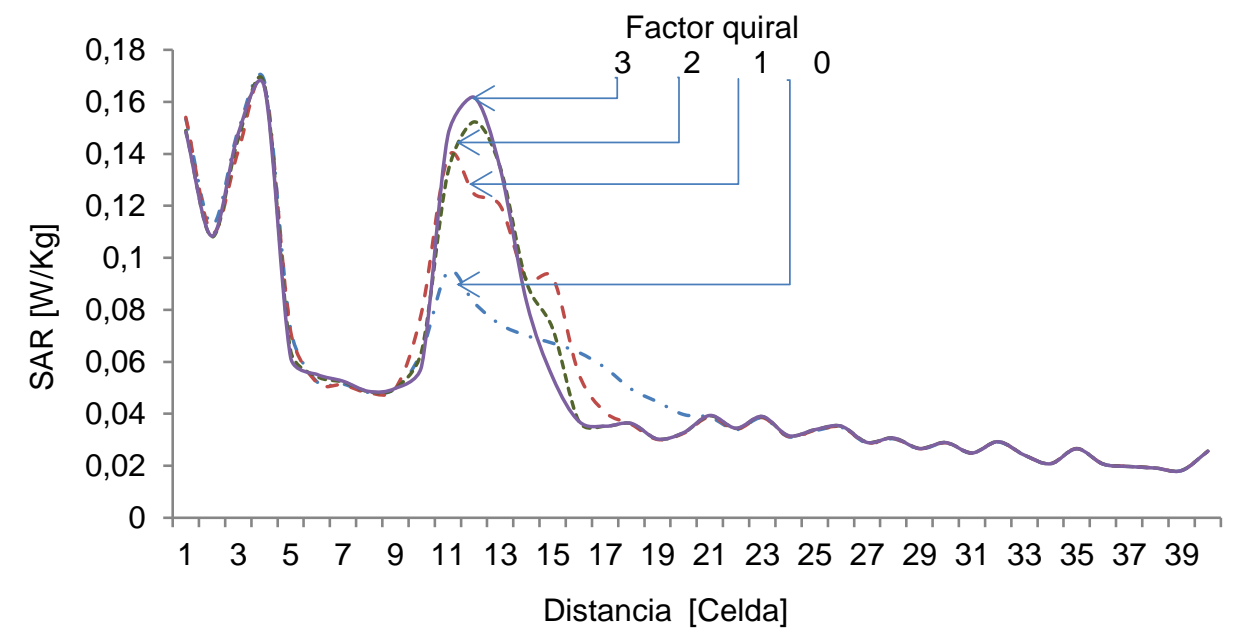

Fig. 5: SAR v/s celdas, con factor quiral variable, para una frecuencia de $2,4 \mathrm{GHz}$.

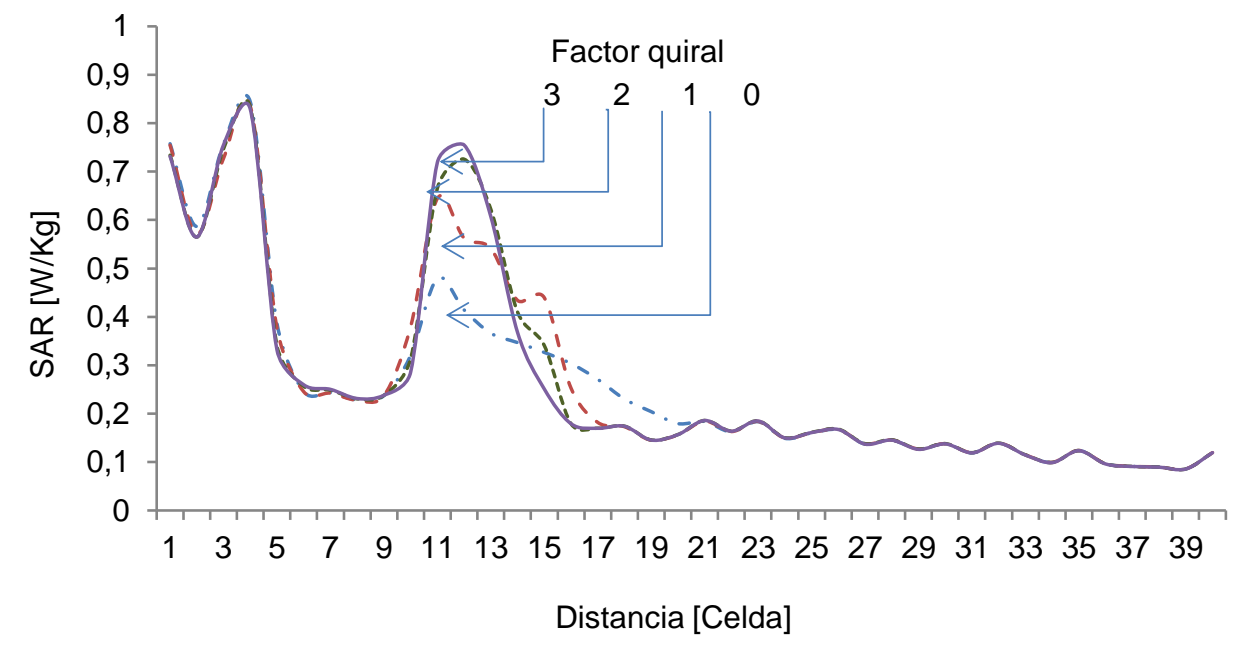

Fig. 6: SAR v/s celdas, con factor quiral variable, para una frecuencia de 5,2 GHz.

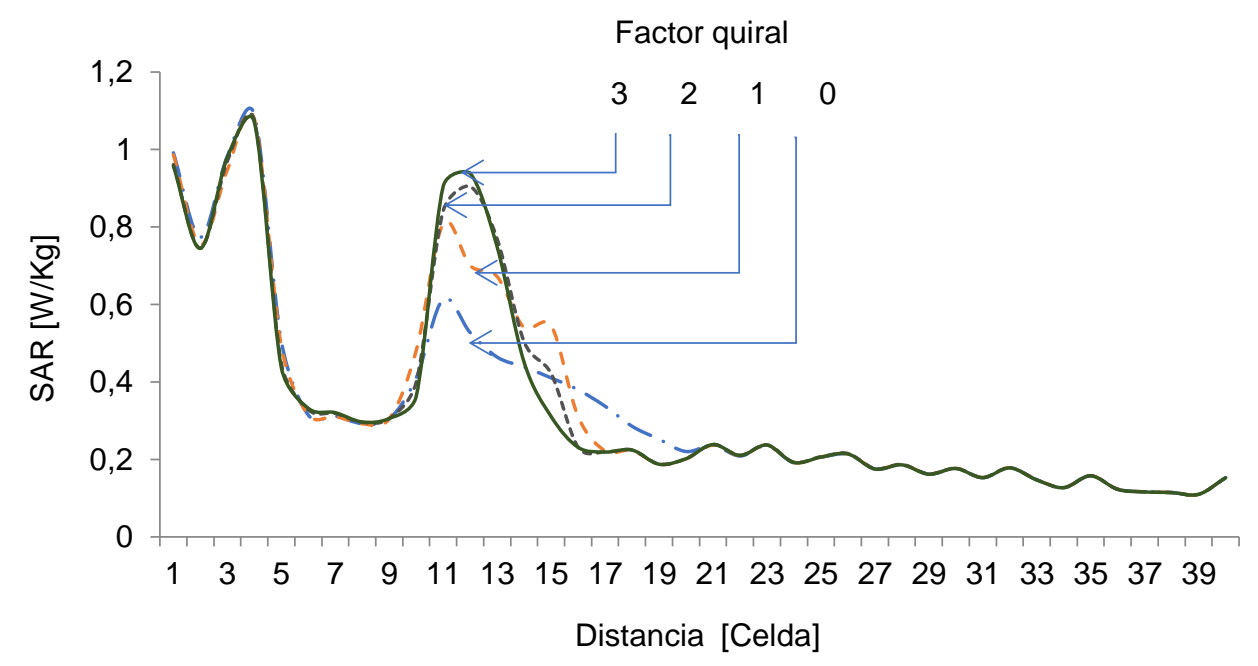

Fig. 7: SAR v/s celdas, con factor quiral variable, para una frecuencia de $5,8 \mathrm{GHz}$.

Finalmente, presentamos en la figura 8 un análisis de los resultados del SAR en función de la frecuencia, es decir de las microondas de los sistemas WiFi y Wi-Max. Se puede observar un aumento paulatino del SAR a medida que aumenta el factor quiral, cualquiera sea la frecuencia de trabajo. Además, en las frecuencias más altas, al pasar de $5,2 \mathrm{GHz}$ a $5,8 \mathrm{GHz}$, el SAR disminuye. Este efecto es más pronunciado para valores más altos del factor quiral. Esta respuesta corresponde a un efecto skin normal. 


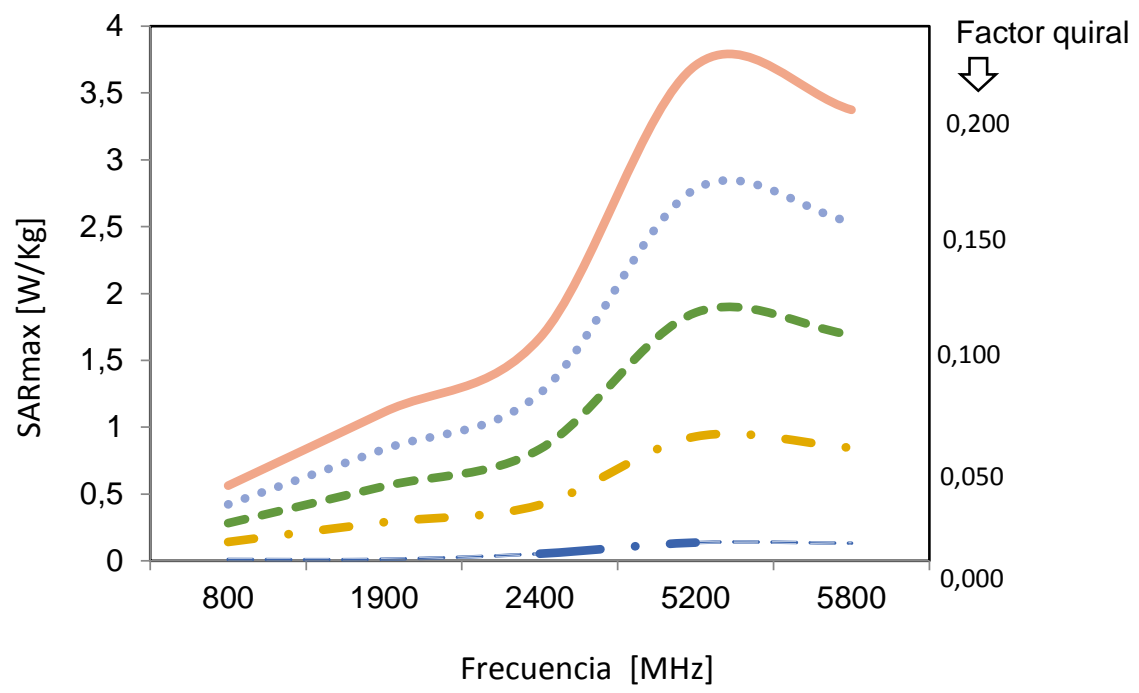

Fig. 8: SAR v/s frecuencia, con factor quiral variable.

\section{DISCUSIÓN FINAL}

Nuestro trabajo ha presentado un modelo bioplasmático quiral de la cabeza humana, el que permite la determinación y evaluación de la absorción inducida por la radiación de los sistemas inalámbricos. Se demostró que el uso de un modelo estándar de sistema inalámbrico, combinado con un modelo real de la cabeza humana, derivado de la resonancia magnética de las imágenes, permite la determinación exacta de los campos inducidos cuando se considera la quiralidad cerebral.

Para las simulaciones de los campos electromagnéticos y del correspondiente SAR, se usaron la plataforma Matlab y el algoritmo FDTD con quiralidad, este último obtenido de las ecuaciones (5) - (10). Los resultados que se lograron corresponden a los sistemas Wi-Fi operando en 2,4 GHz y Wi-Max, operando en 5,2 y 5,8 GHz. Las simulaciones se realizaron de manera sistemática para la capa 35 y los resultados muestran la evolución del SAR, en función del factor quiral y de la frecuencia de trabajo.

\section{CONCLUSIONES}

De acuerdo al trabajo presentado y a los resultados obtenidos, se pueden plantear las siguientes conclusiones principales:

1. El modelo bioplasmático quiral de la cabeza humana permite determinar y evaluar, con mayor precisión, la absorción inducida por la radiación de los sistemas inalámbricos WiFi y Wi-Max, asociados a los sistemas $3 G$ y $4 G$.

2. La magnitud del SAR, determinada a través del modelo de electrodinámica quiral, es mayor que los resultados obtenidos con los modelos clásicos.

3. La absorción de la radiación de microondas aumenta inicialmente con la frecuencia para posteriormente disminuir debido al efecto skin.

\section{AGRADECIMIENTOS}

Este trabajo se ha desarrollado con el apoyo del Programa de Magister en Telecomunicaciones de la Universidad de Tarapacá Arica y financiado a través del Proyecto de Investigación UTA 8731.

\section{REFERENCIAS}

Baliatsas, C., Van Camp I. y otros cuatro autores, Non-Specific Physical Symptoms and Electromagnetic Field Exposure in the General Population: Can We Get More Specific? A Systematic Review, https://doi.org/10.1016/j.envint.2011.12.002, Environment International,41, 15-28 (2012). 
Cardis, E., Deltour I. y otros cuarenta y seis autores, Brain Tumour Risk in Relation to Mobile Telephone Use: Results of the Interphone International Case-Control Study, https://doi.org/10.1093/lje/Dyq079, Int. J. of Epidemiology, 39(3), 675694 (2010).

Chiaramello, E., Bonato M. y otros cinco autores, Radio Frequency Electromagnetic Fields Exposure Assessment in Indoor Environments: A Review. https://doi.org/10.3390/ijerph16060955, Int. J. of Environ. Resarch and Public Health 16(6),129 (2019).

Chiu, C.T., Chang Y.H. y otros tres autores, Mobile Phone Use and Health Symptoms in Children, https://doi.org/10.1016/j.jfma.2014.07.002, Journal of the Formosan Medical Association, 114, 598-604 (2015).

Cho, K.S., y Lee J.M., Influence of Smartphone Addiction Proneness of Young Children on Problematic Behaviors and Emotional Intelligence: Mediating Self-assessment Effects of Parents Using Smartphones, https://doi.org/10.1016/j.chb.2016.09.063, Computers in Human Behavior, 66, 303-311 (2017).

Espinosa, P., Zamorano M. y Torres-Silva H., Campos Paralelos en Medios Quirales, https://doi.org/10.4067/S071807642011000400007, Información Tecnológica, 22(4), 51-58 (2011).

Ferikoğlu, A., Çerezci O. y otros dos autores, Electromagnetic Absorption Rate in a Multilayer Human Tissue Model Exposed to Base-Station Radiation Using Transmission Line Analysis, https://doi.org/10.1109/LAWP.2014.2321283, IEEE Antennas And Wireless Propagation Letters,3, 903-906 (2014).

Feychting, M., Mobile Phones Radiofrequency Fields, and Health Effects in Children - Epidemiological Studies, https://doi.org/10.1016/j.pbiomolbio.2011.09.016, Progress in Biophysics and Molecular Biology, 107, $343-348$ (2011).

Ghandi, M., Yes, the Children are more Exposed to Radiofrequency Energy from Mobile Telephones than Adults, https://doi.org/10.1109/ACCESS.2015.2438782, IEEE Access, 3, 985-988 (2015).

Gonzalez-Rubio, J., Arribas E. y otros dos autores, Radiofrequency Electromagnetic Fields and Some Cancers of Unknown Etiology: An Ecological Study, Science of the Total Environment, 599-600, 834-843 (2017).

Hameroff, S. y Penrose R., Orchestrated Objective Reduction of Quantum Coherence in Brain Microtubules: The "Orch Or" Model for Consciousness, Toward a Science of Consciousness, 507-540 (1996).

ITU International Telecommunication Union, ICT Facts and Figures, 1-8, Geneva, Switzerland (2016).

Krayni, A., Novel A., Methodology to Evaluate Uplink Exposure by Personal Devices in Wireless Networks, https://doi.org/10.1109/TEMC.2016.2524543, IEEE Transactions on Electromagnetic Compatibility, 58 (3), $896-906$ (2016).

Lin, J.C., Carcinogenic Effect of Wireless Communication Radiation in Rodents, Advances in Electromagnetic Fields in Living Systems, $1^{\circ}$ Ed., Springer, 5, 35-82, New York, USA (2009).

Makris, N., Angelone L. y otros cuatro autores, MRI-Based Anatomical Model of the Human Head for Specific Absorption Rate Mapping, https://doi.org/10.1007/s11517-008-0414-z, Medical\& Biological Eng. \& Computing, 46(12), 1239-1251 (2008).

Martínez, M., Martin A., Anguiano M. y Villar R., Comparison of FDTD-Calculated Specific Absorption Rate in Adults and Children when Using a Mobile Phone at 900 and 1800 MHz, Physics in Medicine \& Biology, 49 (2) ,345-354 (2004).

Miyakoshi, J., Tonomura H. y otros tres autores, Effects of Exposure to $5.8 \mathrm{GHz}$ Electromagnetic Field on Micronucleus Formation, DNA Strand Breaks, and Heat Shock Protein Expressions in Cells Derived From Human Eye, https://doi.org/10.1109/TNB.2019.2905491, IEEE Transactions on Nanobioscience, 18(2), 257-260 (2019).

Moore, S.M., McIntosh R.L. y otros dos autores, Modeling the Effect of Adverse Environmental Conditions and Clothing on Temperature Rise in a Human Body Exposed to Radio Frequency Electromagnetic Fields, https://doi.org/10.1109/TBME.2014.2362517, IEEE Transactions on Biomedical Engineering, 62 (2), $627-637$ (2015).

Morris, R. D., Morgan L. LL. y Davis D., Children Absorb Higher Doses of Radio Frequency Electromagnetic Radiation From Mobile Phones Than Adults, https://doi.org/10.1109/ACCESS.2015.2478701, IEEE Access, 3, $2379-2787$ (2015).

Rashed, E., Gomez-Tames J. y Hirata A., Human Head Skin Thickness Modeling for Electromagnetic Dosimetry, https://doi.org/10.1109/ACCESS.2019.2904743, IEEE Access, 7, 46176-46186 (2019).

Sage C., Carpenter D.O. y otros veintinueve autores, A Rationale for Biologically-Based Public Exposure Standards for Electromagnetic Fields ELF and RF, Biolnitiative2012, 1-26, Santa Mónica, USA (2012).

Taflove, A. y Hagness S.C., Mur Finite-Difference Scheme, Computational Electrodynamics: The Finite-Difference Time Domain Method. Artech House. $3^{\text {a }}$ Ed., 6, 240-243, Illinois, USA (2005).

Torres, H. y Zamorano M., SAR Simulation for Chiral Waves in Head Model, Rev. Fac. Ing.-Univ. Tarapacá, 11 (1), $3-11$ (2003).

Torres, H. y Zamorano M., Chiral Multiphoton Absorption and Inverse Skin Effect in Wlan Systems, Rev. Fac. Ing-Univ Tarapacá, 13(3) ,122-130 (2005).

WHO/IARC, Agents Classified by the IARC Monographs, 1-37 (2018).

Wyde, M., Cesta M: y otros doce autores, Report of Partial Findings from the National Toxicology Program Carcinogenesis Studies of Cell Phone Radiofrequency Radiation in Hsd, https://doi.org/10.1101/055699, BioRxiv. 1-89 (2016). 